\title{
Otimização do Streaming de Vídeo utilizando a informação de Consumo de Energia
}

\author{
Diego José de Sousa Gouveia ${ }^{1}$, Demóstenes Zegarra Rodriguez ${ }^{1}$ \\ ${ }^{1}$ Departamento de Ciência da Computação - Universidade Federal de Lavras (UFLA) \\ Caixa Postal 3.037 - 37.200-000 - Lavras - MG - Brasil \\ diegosousa.stecomputacao.ufla.br, demostenes.zegarraedcc.ufla.br
}

\begin{abstract}
Multimedia streaming applications have grown immensely in recent years and have been studied in order to ensure a good QoE for the user. However, few works are concerned with the energy consumption and the characteristics of multimedia content in a deep way, which makes the energy consumption by these contents an obscure area. In this article we present a mathematical model that suggests how the consumption in Ah of a video is in function of its parameters. The proposed model was an exponential archetype, being very close to the real model, describing which properties have the most influence on consumption and should be applied to videos with constant bitrate.
\end{abstract}

Resumo. As aplicações de streaming multimídia têm crescido imensamente nos últimos anos e vêm sendo estudadas com a finalidade de garantir uma boa QoE para o usuário. Entretanto, poucos trabalhos preocupam-se com o consumo de energia e com as características do conteúdo multimídia de forma profunda, o que torna o consumo energético por parte desses conteúdos uma área obscura. Neste artigo apresenta-se um modelo matemático que sugere como é o consumo em Ah de um vídeo em função de seus parâmetros. $O$ modelo proposto foi um arquétipo exponencial, mostrando-se bem próximo do modelo real, descrevendo quais propriedades têm mais influência no consumo, devendo ser aplicado aos vídeos com taxa de bits constante.

\section{Introdução}

O número de fornecedores do serviço de streaming de vídeo está crescendo cada vez mais em todo o mundo, principalmente na plataforma mobile. Em 2014 cerca de 64\% do tráfego na internet mundial consistia de conteúdo de vídeo, e estudos recentes estimam que em 2019 esse número chegará a 80\% [networking Index 2016]. A popularização desse tipo de serviço cresceu tanto que no início de 2017 a Netflix conseguiu superar a marca de 100 milhões de usuários em todo o mundo [Netflix 2017]. Além da Netflix, outros serviços de streaming multimídia como Vimeo, Dailymotion e YouTube contribuem, e muito, para esse enorme aumento no consumo de vídeo. $\mathrm{O}$ YouTube, por exemplo, conta com mais de um bilhão de usuários, quase um terço dos usuários da internet e, a cada dia, as pessoas assistem a milhões de horas de vídeo, gerando bilhões de visualizações. Além disso, mais de $50 \%$ das visualizações do YouTube são feitas em dispositivos móveis [YouTube 2017].

O usuário final, ao utilizar os serviços de streaming, deseja assistir o seu conteúdo de interesse com a maior qualidade e fidelidade possível, além de ter uma experiência positiva, seja ele acessado por meio de um desktop, mobile, ou qualquer 
outro. A experiência pode ser entendida como a sensação de percepções, a qual envolve sentimentos, percepção sensorial e conceitos, que ocorrem em uma situação particular de referência [Möller e Raake 2014]. Entre os fatores que levam à uma experiência positiva e a um alto grau de aceitação de um serviço destacam-se a alta performance, a qualidade de transmissão dos dados e a usabilidade [Möller e Raake 2014]. Para indicar a percepção e a experiência do usuário, utiliza-se o conceito de Quality of Experience (QoE). Esse termo pode ser definido como o grau de prazer ou insatisfação de uma pessoa, cuja o processo de experimentação envolve uma aplicação, serviço ou sistema. A QoE resulta da avaliação da pessoa sobre o cumprimento de suas expectativas e necessidades, com respeito à utilidade e/ou diversão relacionadas ao seu contexto, à sua personalidade e ao seu estado atual [Möller e Raake 2014].

Existem alguns fatores que influenciam a QoE. Esses fatores são denominados Fatores de Influência (Influence Factors-IFs) e são quaisquer características de usuário, sistema, serviço, aplicação ou contexto, cujo estado atual ou configuração possa ter influência na QoE para o usuário [Brunnström et al. 2013]. Esses fatores podem ser divididos em Fatores de Influência Humana (Human Influence Factors-HIFs), Fatores de Influência do sistema (System Influence Factors-SIFs) e Fatores de Influência de Contexto (Context Influence Factors-CIFs) [Brunnström et al. 2013]. Todos são importantes, mas os Fatores de Influência de Sistema são de grande relevância no cenário de streaming de vídeo, visto que se referem às propriedades e características que determinam a qualidade técnica de uma aplicação ou serviço. Os SIFs podem estar relacionados a vários aspectos: ao conteúdo, já que conteúdos diferentes exigem propriedades diferentes do sistema; a mídia, pois configurações de vídeo como codificação, resolução, frame rate entre outros se diferem de um vídeo para outro; a rede, pois a largura de banda, atrasos, variações, perdas e a vazão diferem de usuário para usuário; e ao dispositivo, porque os sistemas finais e suas características também variam muito, sendo que todos estes citados acima afetam diretamente a QoE [Brunnström et al. 2013].

Pode-se dizer que um serviço de streaming de vídeo que não atende aos conceitos de usabilidade e não se atenta aos Fatores de Influência, dentre tantos outros, não fornece ao usuário uma boa QoE.

Como o número de dispositivos móveis passou de 7 bilhões em 2015 e com o grande consumo de vídeo por parte dos usuários da rede mundial de computadores, principalmente através de mobiles, torna-se necessário garantir ao usuário a melhor QoE possível. Entre um dos fatores que contribuem criticamente para isso, está o tempo de vida da bateria do smartphone [Power 2012]. Portanto, é essencial que o streaming de vídeo em mobiles não forneça somente uma boa experiência ao assistir algum vídeo, mas também evite o consumo de energia excessiva [Hoque et al. 2012].

Esse é um dos grandes problemas em dispositivos móveis, pois é complicado manter um serviço com toda a sua qualidade sem prejudicar o consumo da bateria, e é ainda mais dificil achar o trade-off entre qualidade de vídeo e consumo de energia da bateria.

Outros fatores como o tipo de transferência de dados da rede wireless, configurações de hardware e software do dispositivo e os protocolos de comunicação utilizados afetam o consumo do aparelho, ou seja, dependendo dos fatores vistos acima, 
um aplicativo pode fornecer, ou não, uma melhor qualidade de experiência (QoE) para o usuário [Reiter et al. 2014].

Recentemente, várias pesquisas têm sido desenvolvidas a fim de entender e avaliar como o streaming de vídeo afeta o consumo da bateria de smartphones. [Hoque et al. 2012] e [Hoque et al. 2015] analisaram as técnicas de streaming mais utilizadas e chegaram a conclusões a respeito de qual técnica consome mais energia. Entretanto, esses autores não abordaram a fundo como os parâmetros de vídeo afetam o consumo .

Nesse contexto, visando melhorar e personalizar a experiência do usuário, o objetivo principal deste trabalho é conseguir medir com exatidão quais parâmetros de vídeo mais influenciam no consumo da bateria de dispositivos móveis e assim, achar um modelo matemático que expresse o consumo em função desses parâmetros.

O restante do artigo está organizado da seguinte forma, os trabalhos relacionados são apresentados na Seção 2. Na Seção 3 são apresentados os conceitos teóricos dos fatores de influência do sistema e os principais parâmetros de vídeos analisados no presente trabalho. Apresenta-se na Seção 4 a forma como foi realizado o estudo e quais métodos foram utilizados para avaliar e comparar os modelos propostos. A Seção 5 descreve os resultados obtidos com a pesquisa, apresenta o modelo e mostra as limitações do trabalho. Por fim, são apresentadas as Seções 6 e 7, onde são feitas as considerações finais, discutindo-se sobre trabalhos futuros, e os agradecimentos, respectivamente.

\section{Trabalhos relacionados}

O presente trabalho está inserido entre a área de entrega de conteúdo multimídia e a área de otimização de consumo de energia, tendo esta última maior destaque. A forma como um conteúdo é entregue ao usuário afeta o consumo de energia e, consequentemente, a QoE do usuário.

Nesse ramo de entrega de conteúdo, [da Silva et al. 2015] focaram na detecção de streamers em redes BitTorrent, notando que aplicações streaming sobre essas redes podem causar prejuízos sobre o desempenho geral. Segundo [da Silva et al. 2015], os usuários sofrem de dois problemas: falta de escalabilidade relacionada à arquitetura cliente/servidor e acervo limitado de algumas aplicações streaming. Para resolver isso, ainda segundo [da Silva et al. 2015], sistemas P2PTV podem ser utilizadas. Nesses sistemas, os participantes não assistem à mídia somente, mas também transmitem dados para os demais participantes da rede. Essas soluções, embora boas, não se tornaram populares.

Outro estudo que buscou a melhora na QoE do usuário em relação a entrega de conteúdo de vídeo foi o trabalho realizado por [Coelho 2015]. Entretanto, neste trabalho, para atingir o objetivo, foram consideradas somente fatores relacionados à rede do usuário e à técnica de DASH. Esta técnica consiste em entregar vídeo ao usuário de uma forma adaptativa, embasando-se na largura de banda disponível no momento da requisição. Para que isso seja possível, o arquivo de vídeo é cortado em pequenos pedaços com diferentes resoluções, permitindo o download dinâmico sem atrapalhar o conteúdo que já foi baixado, não importando que parte do vídeo está sendo assistida enquanto o resto do vídeo está sendo baixado [Michalos et al. 2012]. 
Já na parte de consumo de energia, [Hoque et al. 2012] observaram que o container do vídeo influencia o consumo, embora não saibam exatamente a causa. Então um vídeo codificado em mp4 terá um consumo diferente de um vídeo codificado em 3gpp, por exemplo. Ademais, o consumo pode ser afetado por características do aparelho, como por exemplo, o tamanho e o tipo do display. Então, os fatores de influência do sistema relacionados ao dispositivo, que serão abordados na seção seguinte, interferem não somente na QoE do usuário, mas também no próprio consumo de energia, visto que características do smartphone se enquadram nesse tipo de fator de influência. Outra importante observação realizada, foi que o consumo eficiente de energia por parte do usuário depende da forma como ele assiste os vídeos. Se o usuário assiste um vídeo em sua totalidade de duração, uma determinada técnica de streaming pode ser melhor, enquanto que, se o usuário assiste somente parte de um vídeo, outras técnicas consumiriam menor quantidade de energia.

Ainda nesse sentido, [Hoque et al. 2015] analisaram o consumo de energia em diferentes smartphones com diferentes formas de entrega de streaming de vídeo e observaram que a qualidade do vídeo, isto é, a resolução, aparentemente não tem grande impacto no consumo de energia, pois em alguns casos que eles testaram, mesmo dobrando a resolução do vídeo, o aumento no consumo foi relativamente pequeno. Além disso, [Hoque et al. 2015] observaram que a plataforma influencia na técnica de streaming escolhida pelo provedor do vídeo, ou seja, dependendo do sistema operacional do smartphone uma técnica de streaming pode ser escolhida em detrimento de outra.

Embora os dois últimos trabalhos citados anteriormente tenham descoberto a melhor forma de streaming em determinadas situações e tenham focado na redução do consumo de energia, nenhum deles aprofundou-se nas características intrínsecas aos vídeos que poderiam afetar o consumo diretamente e, portanto, não mostraram como os parâmetros de vídeo influenciam a drenagem de energia. Por isso, de acordo com o nosso conhecimento, não existe nenhum trabalho na literatura que trata o consumo de energia durante um streaming de vídeo com base em propriedades do próprio vídeo, nem existe atualmente um modelo matemático que expresse tal consumo. Portanto, o presente trabalho se mostra até o momento como o único trabalho que visa modelar o consumo de um dado vídeo em função de seus atributos.

\section{Fundamentação Teórica}

\subsection{Fatores de influência do Sistema}

Vários fatores influenciam na qualidade de um vídeo, como já foi dito anteriormente, e os fatores que mais se destacam são aqueles relacionados ao sistema. Esses fatores podem ser divididos em quatro categorias: Fatores de influência do sistema relacionados ao conteúdo (Content-related System IFs), Fatores de influência do sistema relacionados à mídia (Media-related System IFs), Fatores de influência do sistema relacionados à rede (Network-related System IFs) e Fatores de influência relacionados ao dispositivo (Device-related System IFs).

Os fatores de influência do sistema relacionados ao conteúdo dizem respeito ao próprio conteúdo e seu tipo, tendo grande influência sobre a QoE geral do sistema. No caso de vídeo, por exemplo, a quantidade de detalhes assim como a quantidade de 
movimentação além da profundidade de bits são aspectos que influenciam a qualidade e o conforto ao assistir um vídeo [Möller e Raake 2014].

Já os fatores de influência do sistema relacionados à mídia se referem às características de configuração da mídia, como a codificação, resolução, taxa de amostragem, taxa de quadros, sincronização de mídia, método de compressão, entre outros. Esses fatores estão inter-relacionados com os fatores de influência do sistema relacionados ao conteúdo [Möller e Raake 2014].

Além dos citados acima, têm-se os fatores de influência do sistema relacionados à rede, que são inerentes à transmissão de dados sobre uma rede. As principais características de rede são largura de banda, delays, variações, taxa de perdas e de erros, vazão entre outros [Möller e Raake 2014].

Por último, têm-se os fatores de influência do sistema relacionados ao dispositivo. Este refere-se ao sistema final do usuário. Características como tamanho do display, profundidade de cor, resolução do display, mecanismos de gerenciamento de energia, dentre outros fazem parte do escopo desse tipo de fator [Möller e Raake 2014].

\subsection{Parâmetros que influenciam a qualidade e consumo de energia de vídeo}

Um vídeo possui muitas propriedades objetivas que the confere maior qualidade, maior tamanho em bytes e outras peculiaridades. Tais propriedades podem ser vistas como parâmetros, os quais afetam diretamente a qualidade e o consumo de energia durante a visualização de um determinado vídeo. Ao variar-se determinado parâmetro, é possível obter redução no consumo de energia em troca de um decréscimo na qualidade. Para que seja possível aumentar ou diminuir determinado parâmetro a fim de atingir esse objetivo, é necessário descobrir quais parâmetros afetam com mais intensidade o consumo de energia.

Os principais parâmetros que foram analisados neste trabalho foram a resolução de vídeo (width $x$ height), a taxa de quadros (fps), a taxa de bits (Mbps), a informação espacial $(S I)$ e temporal $(T I)$ de cada vídeo, calculadas a partir de cada frame, o desvio padrão da informação espacial $(S D S I)$ e temporal $(S D T I)$ e a complexidade da cena $(o)$.

A resolução de vídeo constitui-se do número de pixels de largura (width) e de altura (height) do frame. Quanto maior o número de um desses dois parâmetros, maior será o tamanho do frame em relação a quantidade de pixels desenhados na tela, já que o número total de pixels será width $x$ height. Quanto maior a resolução, maior será o consumo de energia, visto que claramente gasta-se mais energia para desenhar uma quantidade maior de pixels na tela.

Já a taxa de quadros $(f p s)$ diz respeito à quantas amostras de frame de uma dada resolução são codificadas em um segundo de vídeo. Aumentando-se este parâmetro, aumentará também a quantidade total de frames do vídeo, o que fará com que o consumo também aumente, visto que mais frames terão de ser processados durante um mesmo intervalo de tempo do vídeo. Além disso, o tamanho do arquivo também será maior.

A taxa de bits, dada em Mbps, representa o número de bits que são processados em uma unidade de tempo. Quanto maior a taxa de bits, melhor é a qualidade do frame e maior é o tamanho do arquivo. Essa taxa pode ser constante ou variável, sendo que, uma taxa variável irá produzir um frame com melhor qualidade em várias aplicações. 
Neste trabalho, no entanto, os vídeos analisados foram codificados à uma taxa de bits constante, com pequenas variações atribuídas à própria forma de codificação, devido ao fato de ser importante manter as características dos vídeos uniformes, a fim de avaliar o consumo. Se os vídeos fossem codificados com uma taxa de bits variável, seria impraticável realizar alguma comparação entre os vídeos, já que estariam com um parâmetro diferente e este poderia alterar o resultado das medições.

A informação espacial $(S I)$ indica a quantidade de detalhe espacial de um frame, sendo geralmente maior para cenas mais complexas espacialmente. Esse parâmetro é baseado no filtro Sobel. Cada frame de vídeo (plano de luminância) no tempo $n\left(F_{n}\right)$ é filtrado com o filtro Sobel $\left[\operatorname{Sobel}\left(F_{n}\right)\right]$. O desvio padrão sobre os pixels $\left(\right.$ std $\left._{\text {space }}\right) \mathrm{em}$ cada frame filtrado com o filtro Sobel é então computado. Essa operação é repetida para cada frame do vídeo resultando em uma série temporal de informações espaciais da cena. O máximo valor nessa série é usado para representar a SI da cena [Installations e Lines 1999]. Esse parâmetro pode ser representado pela equação (1).

$$
S I=\max _{\text {time }}\left\{\operatorname{std}_{\text {space }}\left[\operatorname{Sobel}\left(F_{n}\right)\right]\right\}
$$

Já a informação temporal (TI) indica a quantidade de mudanças temporais de uma sequência de vídeo. Esse parâmetro também é baseado em um plano de luminância e calcula a movimentação. A movimentação é considerada como a diferença entre os valores dos pixels numa mesma posição espacial em frames consecutivos. $M_{n}(i, j)$ como uma função do tempo $(n)$ é definida pela equação (2) [Installations e Lines 1999].

$$
M_{n}(i, j)=F_{n}(i, j)-F_{n-1}(i, j)
$$

Na equação $(2), F_{n}(i, j)$ é o pixel na $i$-ésima linha e $j$-ésima coluna do $n$-ésimo frame no tempo. A informação temporal é então computada como o máximo tempo ( max $_{\text {time }}$ ) do desvio padrão espacial $\left(s t d_{\text {space }}\right)$ da $M_{n}(i, j)$, para todo $i$ e $j$. O parâmetro $T I$ pode então ser obtido a partir da equação (3) [Installations e Lines 1999].

$$
T I=\max _{\text {time }}\left\{\operatorname{std}_{\text {space }}\left[M_{n}(i, j)\right]\right\}
$$

Quanto maior for a movimentação em frames adjacentes, maiores serão os valores de $T I$.

Para ambas propriedades $S I$ e $T I$, quando calculadas para o vídeo inteiro a fim de se conseguir uma única medida de $S I$ e $T I$ para todo o vídeo, devem ser considerados também os desvios padrões da $S I(S D S I)$ e $T I(S D T I)$, pois ao longo de todos os frames de um vídeo, tanto $S I$ quanto $T I$ podem variar consideravelmente. Isto pode ser visto em uma amostra nas figuras 1 e 2 . 


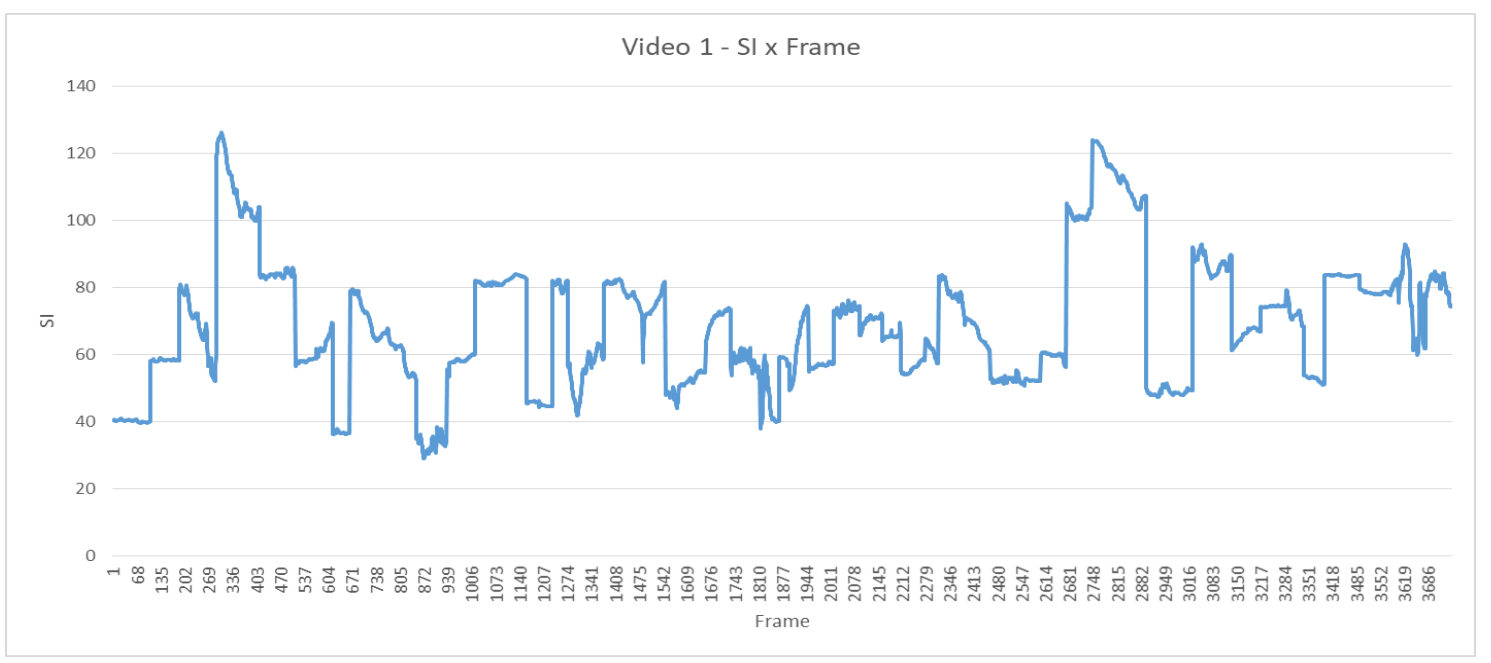

Figura 1. Valores de SI calculados ao longo de um vídeo inteiro

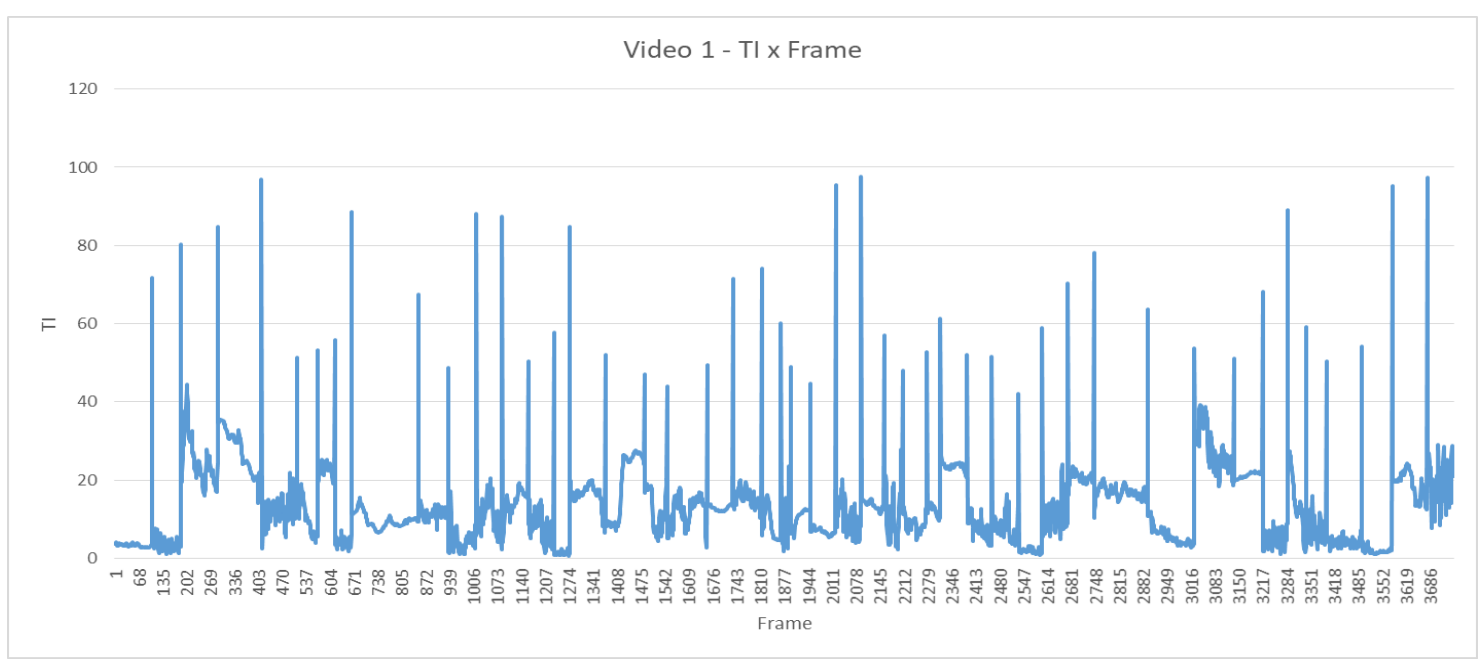

Figura 2. Valores de TI calculados ao longo de um vídeo inteiro

Outro parâmetro que é importante em um vídeo é a complexidade da cena $(o)$. Esse parâmetro é calculado a partir da informação espacial $(S I)$ e temporal $(T I)$ citada anteriormente. Esse método foi apresentado por [Fenimore et al. apud Janowski et al. 2012] e é definido pela equação (4), onde $S I(n)$ e $T I(n)$ representam, respectivamente, a informação espacial e temporal do $n$-ésimo frame.

$$
o=\log _{10}\left(\operatorname{mean}_{n}[S I(n) . T I(n)]\right)
$$

\section{Metodologia}

\subsection{Materiais e procedimentos}

Para a realização da presente pesquisa foram escolhidos 12 vídeos extraídos de um provedor comercial, YouTube, considerando 4 diferentes categorias subjetivas de conteúdo: documentário, música, esporte e notícia. Essas categorias se referem ao tipo de informação trazida pelo vídeo. Por exemplo, um vídeo de futebol poderia ser enquadrado na categoria esporte. Após a seleção dos vídeos, cada um deles foi codificado com duração de 4 minutos, segundo as configurações apresentadas na tabela 1, utilizando-se o software CLI (Command Line Interface) FFmpeg versão 2.8 .11 e o 
container mp4, visto que é um container de vídeo muito utilizado atualmente, totalizando um total de 108 vídeos analisados. Quanto maior fosse a quantidade de vídeos analisados, melhor seria o modelo. Entretanto, a duração dos testes e o número de experiências incrementaria muito. Por isso, julgou-se que a quantidade citada acima seria um valor razoável para que fossem executadas as análises.

As 4 categorias subjetivas citadas acima foram escolhidas a fim de abranger características que podem subjetivamente pertencer a um dado vídeo. Por exemplo, um vídeo de esporte tende a ser mais dinâmico, com mais movimentação. No entanto, nem todo vídeo de esporte é realmente movimentado.

Tabela 1. Configurações de codificação de vídeo

\begin{tabular}{|c|c|c|c|}
\hline Resolução & $15 \mathrm{fps}$ & $30 \mathrm{fps}$ & $60 \mathrm{fps}$ \\
\hline $320 \times 240$ & Configuração 1 & Configuração 2 & Configuração 3 \\
\hline $720 \times 480$ & Configuração 4 & Configuração 5 & Configuração 6 \\
\hline $1920 \times 1080$ & Configuração 7 & Configuração 8 & Configuração 9 \\
\hline
\end{tabular}

A análise do consumo de energia durante a visualização de um determinado vídeo foi feita através do benchmark SiSoftware Sandra Lite Platinum versão 2017.04.24.18. Todas as análises foram realizadas em um notebook com processos em background controlados e uma bateria com capacidade máxima de $35.71 \mathrm{Wh} / 2.48 \mathrm{Ah}$ com 14,4 Volts. Durante todos os experimentos, o notebook estava sem conexão à fonte de alimentação, utilizando somente a energia proveniente da bateria do dispositivo. A escolha de uso de um notebook ao invés de um dispositivo mobile se deu devido ao fato de ser possível controlar, prever e analisar mais facilmente o consumo neste dispositivo, visto que, nesta etapa de análise, os parâmetros que afetam a qualidade e consumo de energia dos vídeos dependem exclusivamente dos vídeos, não sendo, por isso, necessário a análise em dispositivos mobiles nesta etapa.

Todos os dados coletados durante as medições foram registrados e compilados. Dentre as informações fornecidas pelo benchmark após a análise dos dados, o parâmetro para avaliação que melhor representava o consumo do vídeo foi o consumo Ah. Este valor representa o quanto um vídeo consumiu de energia em Amperes/hora.

Considerando este parâmetro, algumas medições foram discrepantes e fugiram muito do padrão medido para uma determinada configuração de vídeo. Para que esses dados de outlier não interferissem nas medições que seriam posteriormente usadas para a criação do modelo matemático, foram calculados novos valores aproximados para esses dados utilizando-se a média aritmética das medições para uma dada configuração de vídeo. Assim, por exemplo, um vídeo com uma resolução 1920x1080 e 60 fps que possuía medição de consumo Ah muito baixo, teve sua medição aproximada para a média das medições dessa mesma configuração de vídeo.

Assim, ao final obteve-se o gráfico exibido na Figura 3. 


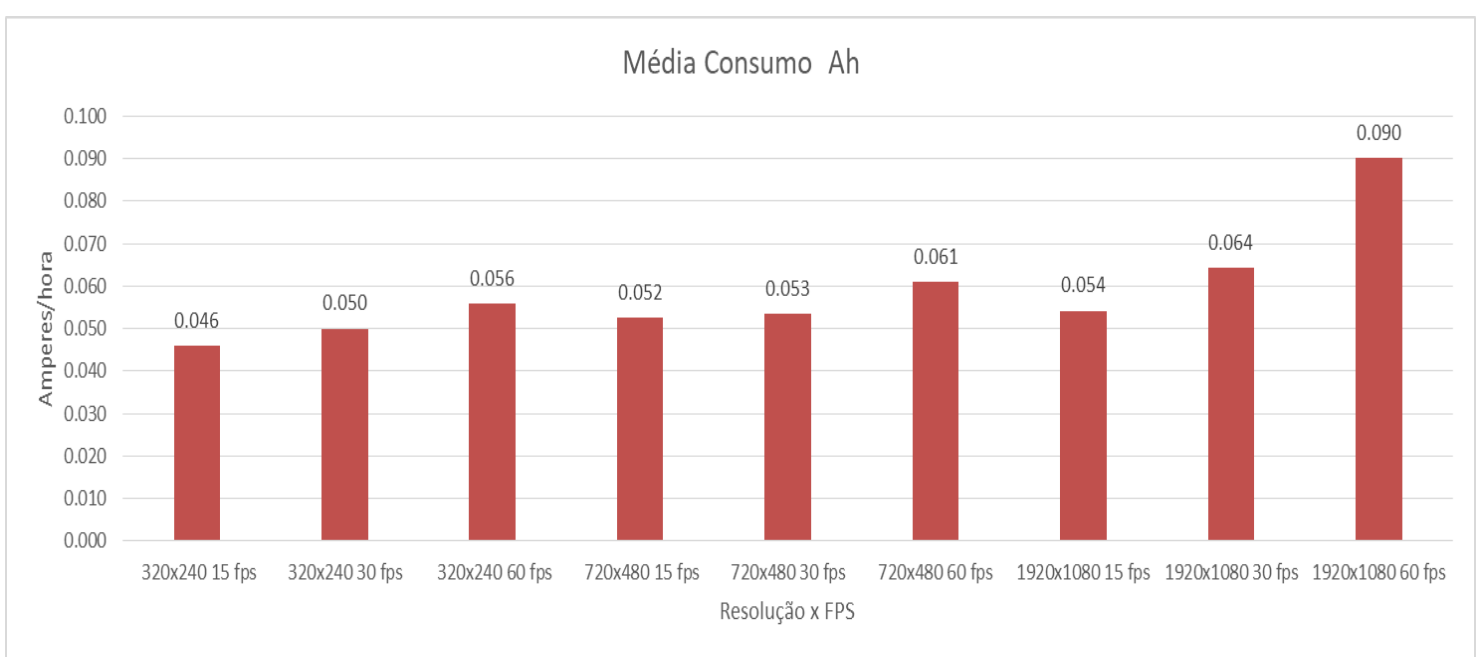

Figura 3. Média de consumo Ah por configuração de vídeo.

De posse das medições, foram propostos dois modelos que representassem o comportamento do consumo de vídeo, um modelo linear da forma $x_{1} p_{1}+x_{2} p_{2}+\cdots+$ $x_{n} p_{n}=A h$, e um modelo exponencial da forma $K e^{\left(x_{1} p_{1}+x_{2} p_{2}+\cdots+x_{n} p_{n}\right)}=A h$, onde $p_{n}$ representa o enésimo parâmetro de vídeo, $A h$ representa o consumo estimado, $x_{n}$ representa os coeficientes encontrados para o modelo e $K$ representa uma constante do modelo exponencial.

Para que fossem calculados os valores de $x_{n}$ dos dois modelos, foi utilizado o método dos mínimos quadrados. Com este método, foi possível determinar os parâmetros do modelo matemático através de valores dessa função em pontos discretos de um intervalo, levando-se em consideração os erros experimentais. Para o caso do modelo exponencial, foi feita a linearização do modelo a fim de utilizar-se do método dos mínimos quadrados. Cada modelo foi calculado levando-se em conta 5 combinações de parâmetros, conforme mostrado na tabela 2 .

Tabela 2. Combinações de parâmetros utilizados para cálculo dos modelos

\begin{tabular}{|c|c|}
\hline Linear & Exponencial \\
\hline width + height $+f p s$ & width + height $+f p s+\ln (K)$ \\
\hline width + height $+f p s+o$ & width + height $+f p s+o+\ln (K)$ \\
\hline$S I+T I+m b p s+w i d t h+h e i g h t+f p s$ & $S I+T I+m b p s+w i d t h+h e i g h t+f p s+\ln (K)$ \\
\hline$S I+S D S I+T I+S D T I+m b p s+w i d t h+h e i g h t+f p s$ & $S I+S D S I+T I+S D T I+m b p s+w i d t h+h e i g h t+f p s+\ln (K)$ \\
\hline$S I+T I+m b p s+w i d t h+h e i g h t+f p s+o$ & $S I+T I+m b p s+w i d t h+h e i g h t+f p s+o+\ln (K)$ \\
\hline
\end{tabular}

Após a resolução do método de mínimos quadrados, realizado pelo software Matlab, foram calculados, para cada modelo com cada combinação de parâmetros, o PCC e a RMSE, a fim de comparar os resultados obtidos e assim identificar qual o melhor possível modelo para representar o consumo de energia durante a visualização de um vídeo de acordo com os parâmetros acima citados.

\subsection{Métricas para avaliar o desempenho da solução proposta}

A fim de determinar o grau de relação entre duas variáveis foi utilizado neste trabalho o Coeficiente de Correlação de Pearson (Pearson Correlation Coefficient - PCC). Este coeficiente de correlação representa o grau de dependência linear entre duas variáveis e 
seu valor está compreendido entre -1 e 1 . Se o valor deste coeficiente for negativo, significa que uma variável diminui enquanto a outra aumenta. Caso contrário, se for positivo, significa que uma variável aumenta quando a outra aumenta. O cálculo do PCC para duas variáveis $\mathrm{x}$ e y pode ser expresso de acordo com a equação (5) [Lee Rodgers apud Zhou et al. 2016].

$$
r_{x y}=\frac{\sum\left(x_{i}-\bar{x}\right) \sum\left(y_{i}-\bar{y}\right)}{\sqrt{\sum\left(x_{i}-\bar{x}\right)^{2}} \sqrt{\sum\left(y_{i}-\bar{y}\right)^{2}}}
$$

Onde,

$\bar{x}=$ média do conjunto de dados da variável $x$

$x_{i}=$ valor da variável $x$ no ponto $i$.

$\bar{y}=$ média do conjunto de dados da variável $y$

$y_{i}=$ valor da variável $y$ no ponto $i$.

Um cálculo do PCC acima de 0.7 é considerado bom, isto é, acima deste valor, existe uma dependência linear muito forte entre as variáveis, aumentando cada vez mais à medida que este valor se aproxima de 1 .

Outra medida utilizada para avaliar os experimentos feitos foi a RMSE (Root Mean Square Error). Essa medida é frequentemente usada para medir a diferença entre valores preditos por um modelo e os valores realmente observados no ambiente que está sendo modelado. Cada diferença individual desses valores são chamados de resíduos, e a RMSE é utilizada para agregar todos esses resíduos em uma única medida. A RMSE para um conjunto $n$ de dados pode ser calculada como mostrado na equação (6) [Chai e Draxler 2014].

$$
R M S E=\sqrt{\frac{\sum_{i=1}^{n}\left(x_{o b s, i}-x_{m o d}, i\right)^{2}}{n}}
$$

Onde, $x_{\text {obs }}$ representa o valor observado e $x_{\text {mod }}$ representa o valor estimado pelo modelo, ambos no instante $i$. Quanto menor o valor da RMSE, melhor é o modelo analisado.

Por fim, mas não menos importante, foi empregado o método dos mínimos quadrados aplicados à solução de sistemas de equações lineares. Segundo [Lima e Sampaio 2009], este método define que, dado um sistema da forma $A x=b$, sem solução por eliminação gaussiana, é possível calcular um vetor $x^{*}$ que é o mais próximo possível da solução do sistema. Este vetor minimiza a norma euclidiana $\|A x-b\|$. Para que isso seja possível, as colunas da matriz $A$ devem ser linearmente independentes.

Teorema: A solução pelo método dos mínimos quadrados para o sistema de equações linear $A x=b$, é definida pelas equações (7) e (8) [Lima e Sampaio 2009].

$$
\begin{gathered}
\left(A^{T} A\right) x=A^{T} b \\
x^{*}=\left(A^{T} A\right)^{-1} A^{T} b
\end{gathered}
$$


2009].

E o erro associado a este método é definido pela equação (9) [Lima e Sampaio

$$
e=\left\|A x^{*}-b\right\|^{2}
$$

\section{Resultados e Discussão}

Para que seja possível melhorar a QoE do usuário quanto à vida útil da bateria durante o streaming de um vídeo, é preciso entender quais parâmetros intrínsecos ao vídeo afetam o consumo de energia e, com a presente pesquisa, isto foi alcançado. A partir da análise feita neste trabalho, foi possível estimar um modelo matemático que sugere como se comporta o consumo em Ah durante a visualização de um vídeo.

$\mathrm{O}$ modelo que apresentou melhor relação com o consumo medido e ficou mais próximo do consumo real foi o modelo exponencial com a combinação de parâmetros $S I+S D S I+T I+S D T I+m b p s+$ width + height $+f p s+\ln (K)$, que apresentou o melhor PCC e melhor RMSE, conforme as tabelas 3 e 4 .

Tabela 3. Coeficiente de Correlação de Pearson dos modelos

\begin{tabular}{|c|c|c|c|c|c|c|c|c|c|}
\hline \multicolumn{10}{|c|}{ Coeficiente de Correlação de Pearson (Estimado x Real) } \\
\hline \multirow{2}{*}{ Linear } & $\begin{array}{c}\text { Linear } \\
\text { SI+TI+SDs }\end{array}$ & $\begin{array}{c}\text { Linear } \\
\text { SI+TI+o }\end{array}$ & $\begin{array}{c}\text { Linear } \\
+o\end{array}$ & $\begin{array}{c}\text { Linear } \\
\text { SI+TI }\end{array}$ & $\begin{array}{c}\text { Exp } \\
\text { SI+TI+SDs }\end{array}$ & $\begin{array}{c}\text { Exp } \\
\text { SI+TI+o }\end{array}$ & Exp o & $\begin{array}{c}\text { Exp } \\
\text { SI+TI }\end{array}$ & Exp \\
\hline 0.7760 & 0.8294 & 0.8265 & 0.8129 & 0.8246 & $\mathbf{0 . 8 4 9 2}$ & 0.8457 & 0.8440 & 0.8442 & 0.8443 \\
\hline
\end{tabular}

Tabela 4. RMSE dos modelos

\begin{tabular}{|c|c|c|c|c|c|c|c|c|c|}
\hline \multicolumn{10}{|c|}{ RMSE } \\
\hline Linear & $\begin{array}{c}\text { Linear } \\
\mathrm{SI}+\mathrm{TI}+\mathrm{SD} \\
\mathrm{s}\end{array}$ & $\begin{array}{c}\text { Linear } \\
\mathrm{SI}+\mathrm{TI}+\mathrm{O}\end{array}$ & $\begin{array}{c}\text { Linear } \\
+\mathrm{o}\end{array}$ & $\begin{array}{l}\text { Linear } \\
\text { SI+TI }\end{array}$ & $\underset{\text { SI+TI+SDs }}{\operatorname{Exp}}$ & $\begin{array}{c}\text { Exp } \\
\text { SI+TI+o }\end{array}$ & Exp o & $\begin{array}{c}\text { Exp } \\
\text { SI+TI }\end{array}$ & Exp \\
\hline 0.0097 & 0.0081 & 0.0082 & 0.0085 & 0.0082 & 0.0077 & 0.0079 & 0.0079 & 0.0078 & 0.0078 \\
\hline
\end{tabular}

Assim, com base nos valores calculados segundo o PCC, o modelo ficou definido conforme a equação (10).

$$
K e^{\left(x_{1} S I+x_{2} S D S I+x_{3} T I+x_{4} S D T I+x_{5} m b p s+x_{6} w i d t h+x_{7} h e i g h t+x_{8} f p s\right)}=A h
$$

Onde,

$$
\begin{aligned}
& x_{1}=-8.7347 e-04, \\
& x_{2}=-5.0349 e-04, \\
& x_{3}=-9.3939 e-04, \\
& x_{4}=0.0076, \\
& x_{5}=0.2003, \\
& x_{6}=0.0194, \\
& x_{7}=-0.0404 \\
& x_{8}=0.0063, \\
& K=0.607380397
\end{aligned}
$$


Como a duração dos vídeos analisados foi de 4 minutos, o modelo da equação (10) somente se aplicaria a vídeos com 4 minutos. Portanto, para a generalização do modelo, é preciso acrescentar a duração como um parâmetro do mesmo. Logo, com base no modelo apresentado acima e na duração dos vídeos analisados, o modelo completo proposto para um vídeo com duração $d$, dada em minutos, pode ser descrito conforme a equação (11).

$$
\frac{d K e^{\left(x_{1} S I+x_{2} S D S I+x_{3} T I+x_{4} S D T I+x_{5} m b p s+x_{6} \text { width }+x_{7} \text { height }+x_{8} f p s\right)}}{4}=A h
$$

Os resultados apresentados, demonstram que é possível modelar o consumo de energia em função dos parâmetros de vídeo. A figura 4 mostra um comparativo entre o modelo matemático encontrado e o modelo real.

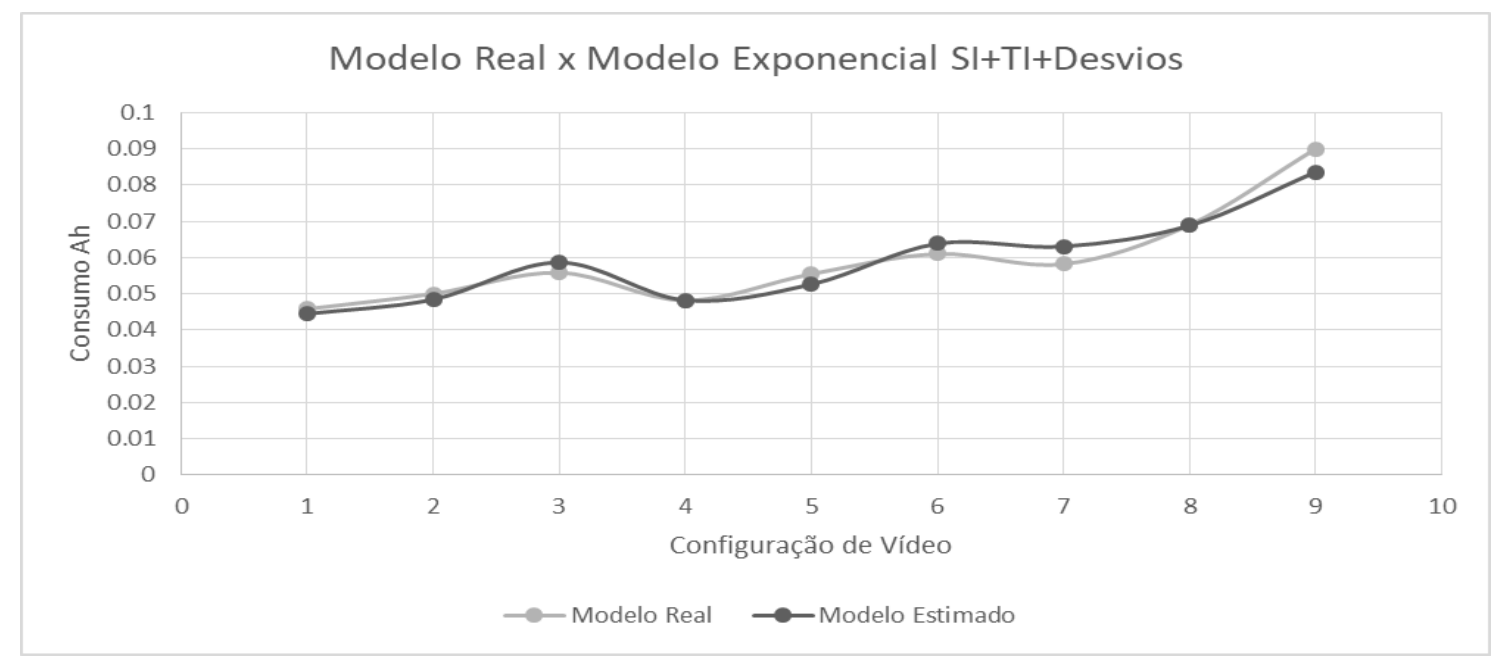

Figura 4. Comparação entre o modelo estimado e o modelo real. Em cinza escuro o modelo estimado e em cinza claro o modelo real.

Este trabalho, comprova o que [Hoque et al. 2015] observaram - a resolução não tem tanto peso no consumo de energia, não sendo tão óbvio observar-se isso. Segundo o modelo apresentado acima, os parâmetros simples que mais influenciam no consumo de energia são a taxa de bits (mbps) e a taxa de quadros (fps). O desvio padrão da informação temporal $(S D T I)$ também se mostrou um fator importante.

É importante que se note que o modelo apresentado somente pode ser aplicado para vídeos codificados com taxa de bits constante e que mesmo assim, devido às pequenas variações mesmo com esse tipo de codificação, o modelo apresentará um pequeno erro nas previsões, que pode ser atenuado ou não de acordo com a influência da duração do vídeo, das características do dispositivo utilizado durante o streaming, do sistema operacional, do mecanismo de gerenciamento de energia do dispositivo entre outros fatores. Em casos reais, dependendo das condições da rede, a taxa de bits de codificação pode variar, bem como outros parâmetros, como em soluções atuais de DASH. Mesmo assim, a proposta apresentada é aplicável nessas condições, sendo necessário fazer um modelo completo definido como a soma das partes, onde em cada parte é necessário aplicar o modelo sobre o trecho de vídeo que contém os parâmetros diferentes do trecho anterior.

Vale destacar ainda que o presente estudo focou no container de vídeo mp4, e por isso, o modelo apresentado acima aplica-se somente a vídeos codificados neste 
container. Outra restrição deste trabalho é que ele foi focado nas propriedades intrínsecas aos vídeos - (Media-related System IFs), e não em outros tipos de fatores de influência do sistema.

\section{Conclusão}

Nesta pesquisa, foi estudado como os parâmetros de vídeo afetam o consumo de energia, visando aumentar a QoE do usuário ao visualizar um vídeo em forma de streaming. $\mathrm{O}$ principal objetivo deste trabalho, que era entender como as características de um dado vídeo influenciam no consumo de energia e gerar um modelo matemático, foi atingido com sucesso. Embora haja limitações, como descrito na seção anterior, o modelo proposto se mostrou bastante próximo ao modelo real. Pode-se dizer que agora, já é possível estimar quanto tempo restante uma bateria tem ao ser usada para reprodução de vídeo.

O principal ganho com este trabalho, é que agora, entendido o comportamento de um vídeo em sua maior parte, é possível criar técnicas de streaming que levem em consideração também o consumo de energia, ao invés somente da largura de banda, como muitas técnicas tratam. Portanto, é possível a partir desta pesquisa, aliar as técnicas de DASH com os parâmetros de vídeo, a fim de fornecer para o usuário a melhor QoE.

Mesmo com os resultados se mostrando bons, entretanto, restam ainda alguns pontos que merecem ser analisados no futuro, principalmente no que diz respeito às medições discrepantes analisadas, isto é, referentes aos dados de outlier. É preciso entender o que causa tais medidas. Outro fator que deve ser posteriormente estudado é a influência da duração do vídeo no consumo de energia, pois não se sabe até o momento se o comportamento da bateria permanece o mesmo durante curtos e longos períodos de tempo de reprodução de um determinado vídeo.

A fim de aumentar a acurácia e precisão, seria bastante interessante que trabalhos futuros, que realizarão medições e experimentos com vídeo acerca deste tema, utilizem técnicas e formas de medição que se utilizam diretamente do hardware. Com tal tipo de medição, ao contrário de se utilizar um benchmark, os erros experimentais provavelmente seriam diminuídos e os resultados poderiam ser melhorados ainda mais. Outro item importante seria um estudo comparativo entre vídeos codificados com containers diferentes, como AVI e 3gpp, por exemplo. Além disso, a fim de melhorar e expandir o modelo proposto, seria de grande valia um estudo em que fossem acrescentados outros tipos de fatores de influência do sistema, pois deixariam o modelo mais completo, representando o cenário real de uma rede.

\section{Agradecimentos}

Este trabalho foi financiado pela Fundação de Amparo à Pesquisa do Estado de Minas Gerias (FAPEMIG).

\section{Referências}

Brunnström, K., Beker, S. A., De Moor, K., Dooms, A., Egger, S., Garcia, M. N., ... \& Lawlor, B. (2013). Qualinet white paper on definitions of quality of experience. 
Chai, T., \& Draxler, R. R. (2014). Root mean square error (RMSE) or mean absolute error (MAE)?-Arguments against avoiding RMSE in the literature. Geoscientific model development, 7(3), 1247-1250.

Coelho, M. D. S. (2015). Estratégia de adaptação de fluxo de vídeo baseada em Fatores de QoE.

da Silva, D. V., Antonio, A. D. A., \& Niterói, R. J. (2015). Detecção de streamers em redes BitTorrent.

Hoque, M. A., Siekkinen, M., Nurminen, J. K., \& Aalto, M. (2012). Investigating streaming techniques and energy efficiency of mobile video services. arXiv preprint arXiv:1209.2855.

Hoque, M. A., Siekkinen, M., Nurminen, J. K., Aalto, M., \& Tarkoma, S. (2015). Mobile multimedia streaming techniques: QoE and energy saving perspective. Pervasive and Mobile Computing, 16, 96-114.

Installations, T., \& Line, L. (1999). Subjective video quality assessment methods for multimedia applications. Networks, 910, 37.

Janowski, L., Romaniak, P., \& Papir, Z. (2012). Content driven QoE assessment for video frame rate and frame resolution reduction. Multimedia tools and applications, 61(3), 769-786.

LIMA, R. D. Q., \& SAMPAIO, R. (2009). Identificação de Parâmetros pelo Método dos Mínimos Quadrados Não Linear. Relatório final da Iniciação Científica. Rio de Janeiro, agosto de.

Michalos, M. G., Kessanidis, S. P., \& Nalmpantis, S. L. (2012). Dynamic Adaptive Streaming over HTTP. Journal of Engineering Science \& Technology Review, 5(2).

Möller, S., \& Raake, A. (Eds.). (2014). Quality of experience: advanced concepts, applications and methods. Springer.

Netflix (2017). Netflix Overview. Acesso em 01 de Agosto de 2017, disponível em Netflix: https:/ir.netflix.com/.

networking Index, C. V. (2016). Forecast and methodology, 2016-2021, white paper. San Jose, CA, USA.

Power, J. D. (2012). US Wireless Smartphone and Traditional Mobile Phone Satisfaction Studies. Press Release.

Reiter, U., Brunnström, K., De Moor, K., Larabi, M. C., Pereira, M., Pinheiro, A., ... \& Zgank, A. (2014). Factors influencing quality of experience. In Quality of experience (pp. 55-72). Springer International Publishing.

YouTube (2017). YouTube para a imprensa. Acesso em 02 de Agosto de 2017, disponível em YouTube: https://www.youtube.com/intl/pt-BR/yt/about/press/.

Zhou, H., Deng, Z., Xia, Y., \& Fu, M. (2016). A new sampling method in particle filter based on Pearson correlation coefficient. Neurocomputing, 216, 208-215. 